


\title{
Identidades y pertenencias como posicionamientos. Un abordaje desde la interseccionalidad
}

\author{
Claudia Arce Cuadros \\ (Flacso, Ecuador)
}

Recibido: $1 / 3 / 2016$

Aprobado: 11/3/2016

\begin{abstract}
Resumen. En este artículo reflexionamos acerca de los términos en los que pueden pensarse las identidades y los sentidos de pertenencia en contextos pluriculturales. Para este fin, examinamos la propuesta de 'cosmopolitismo vernáculo' de Stuart Hall, quien entiende las identidades como puntos de sutura entre discursos y prácticas; es decir, alineaciones entre dos ámbitos: un afuera constitutivo y una interioridad de producción del sujeto. Por otra parte, analizamos la perspectiva de la 'interseccionalidad', que considera que los ejes de estratificación están interconectadamente constituidos los unos a los otros; por tanto, las identidades no se construyen en relación a grupos fijos: clase, etnia, nación, etc., sino como posicionamientos sociales que permitirían representar las múltiples identificaciones operando simultáneamente de manera contingente.
\end{abstract}

Palabras clave: identidad / pertenencia / pluriculturalidad / cosmopolitismo / interseccionalidad

\section{Identity and Belonging as Positionality. An Approach from Intersectionality}

Summary. In this article terms we may use to refer to identities and the meanings of belonging in a multicultural context are considered. For this purpose, we study Stuart Hall's concept of vernacular cosmopolitism, which recognizes identities as points of suture between discourses and practices; that is, alignments between two scopes: a constitutive outside, and interiority produced by the self. On the other hand, we study the intersectionality approach, which considers that axes of social stratification are mutually organized and interconnected; therefore, identities are not built in relation to fixed groups such as class, ethnic group, nation, but as social positionalities letting the representation of multiple identities to operate simultaneously as possible.

Key words: identity / belonging / multicultural / cosmopolitanism / intersectionality 


\section{Introducción}

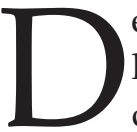
esde los estudios críticos de la migración, en articulación con los estudios culturales y los de género, se coincide en que el sentido de pertenencia puede ir más allá del ámbito nacional y que la noción de identidad se construye de forma transcultural. En estas páginas, a partir de la perspectiva de la interseccionalidad, por una parte, con voces como la de Floya Anthías y Nira Yuval Davis; y por otra parte, con la propuesta de 'cosmopolitismo vernáculo' de Stuart Hall, reflexionamos sobre los términos en los que puede pensarse la configuración de las identidades y los sentidos de la pertenencia en contextos pluriculturales.

En un primer momento examinamos las implicaciones analíticas del planteamiento de 'cosmopolitismo vernáculo' de Stuart Hall como otra manera de entender la pertenencia en contextos multi o pluriculturales, siendo este concepto un intento de reflexionar sobre la relevancia política de afinidades y lealtades múltiples y en conflicto. Si bien, en Political belonging in a world of multiple identities (2002), Hall sugiere el uso estratégico de las identidades múltiples, por ejemplo a través de una 'doble conciencia'; sin embargo, consideramos que no explicita claramente la manera como opera este arreglo de la diferencia (différance) en la identidad; por tanto, revisamos otras obras del autor que permitan entender esta cuestión en el segundo acápite.
Posteriormente, identificamos los principales postulados de la perspectiva interseccional para entender que en los diferentes ejes de estratificación se construyen mutuamente los unos a los otros, de manera que las desigualdades están articuladas y conectadas a las diferencias, y que las identidades se conforman en el entrecruzamiento de diversos clivajes sociales, prácticas y experiencias.

En un cuarto punto examinamos el planteamiento de Floya Anthias respecto al 'posicionamiento translocacional' (translocational posicionality) para pensar las identidades no como adscripciones fijadas a grupos tales como los de género, clase, nacionalidad, etnicidad, sino como posicionamientos que se configuran en la interacción de narrativas hegemónicas que aluden a estos clivajes, y las prácticas de sentido que se constituyen en recursos de acción social. Finalmente, planteamos algunas ideas de cierre retomando los aportes previos sobre lo que representaría pensar las identidades y pertenencias desde posicionamientos que se desplazan continuamente en términos espacio-temporales así como entre divisiones sociales.

\section{'Cosmopolitismo vernáculo'. Otra manera de entender la comunidad y las identidades políticas en contextos pluriculturales}

Stuart Hall está interesado en la definición de comunidad política y de 
pertenencia política más allá del nacionalismo metodológico; es decir, analiza estas cuestiones desde una perspectiva en que las comunidades y las sociedades tienen cada vez más una naturaleza culturalmente múltiple, están compuestas por colectivos que teniendo diferentes orígenes y tradición, y habiendo atravesado fronteras territoriales o espaciales, conviven unas con otras. Se refiere a comunidades y relaciones 'transnacionales', pero bien podemos asociarlas con sociedades 'plurinacionales' como las latinoamericanas en general. En ese contexto, la cuestión del cosmopolitismo se entiende como una forma de articular el significado político de afinidades y lealtades en tensión e impugnación.

El primer punto de una perspectiva cosmopolita que Hall resalta es el del papel central de la cultura en las elecciones significativas y la identidad, pues se trata de la dimensión en la que obtenemos el sentido de la existencia. La pregunta que sigue en un contexto culturalmente diverso es: en torno a qué vocabulario cultural se va a articular la identidad. En contraste con la perspectiva del universalismo liberal, para Hall la cultura es un ámbito abarcativo que contiene una variedad de repertorios discursivos que no son ellos mismos formas puras, intactas, sino que están contaminados unos con otros, pero en relaciones de poder. Se trata "de ver al individuo no simplemente relacionándose con algunos significados culturales, sino dialógica- mente constituido por la existencia del otro" (Hall, 2002, p. 30).

Para este autor, las identidades políticas y todas las identidades son posiciones subjetivas o representaciones que el sujeto asume en el contexto de una interpelación dada; por lo que prefiere los términos de identificación o articulación en lugar de identidad, en tanto esta última evoca unidad, homogeneidad interna $y$, por el contrario, la unidad se construye en torno a lo que queda fuera en el juego del poder y exclusión, como resultado del proceso naturalizado y sobredeterminado de "cierre" (Bhabha, 1994, y Hall, 1993, en Hall, 1996, p. 19).

Uso "identidad" para referirme al punto de encuentro, el punto de sutura entre, por un lado, los discursos y prácticas que intentan "interpelarnos", hablarnos o ponernos en nuestro lugar como sujetos sociales de discursos particulares y, por otro, los procesos que producen subjetividades, que nos construyen como sujetos susceptibles de "decirse". De tal modo, las identidades son puntos de adhesión temporaria a las posiciones subjetivas que nos construyen las prácticas discursivas. (Hall, 1996, p. 20)

Aquí nos interesa el llamado a la diferencia que se hace desde el cosmopolitismo vernáculo en la construcción de las identidades y lo que implica considerar las limitaciones de cualquier y toda cultura o de cualquier y toda identidad para gobernar completamente una sociedad, y la persistencia del reclamo por la dife- 
rencia (Hall, 2002, p. 30). En Political belonging in a world of multiple identities (2002) esta idea está en conexión con las reflexiones sobre la comunidad, lo comunitario, la tradición en la conformación de las comunidades políticas y las identidades ciudadanas. La crítica que hace se dirige hacia el andamiaje del universalismo liberal que, por un lado, concibe un cosmopolitismo aculturado (decultured), en que artificiosamente una aparente neutralidad valorativa acogería todos los contenidos culturales, mientras, por otro, entiende a las comunidades (minorías o mayorías étnicas) fundadas en tradiciones estáticas que han perdido su eficacia como marcos de sentido, y por tal motivo recurren a la vía autoritaria.

La configuración de comunidades políticas desde un cosmopolitismo vernáculo, según Hall, pasa por sociedades que reconocen la importancia de la comunidad y la cultura, y -al mismo tiempo- reconocen el límite liberal del comunitarismo, es decir, el derecho de los individuos de decir no a los aspectos de la cultura que consideren que deben salir, manteniendo al mismo tiempo la viabilidad de la comunidad; además, dice, se trata de un cosmopolitismo conocido, pues ha sido la manera usual de vincularnos con nuestras culturas familiares (Hall, 2002, p. 30).

Estamos en esa situación abierta absoluta y extremadamente difícil de caracterizar, donde las comunidades importan, pero no podrían ser emancipadas en su propio derecho, porque eso simplemente significaría que co- miencen a ejercer de policía sobre todos. Desde el interior de estas comunidades muchas personas actualmente mantienen esa doble conciencia que cualquiera esperaría. (Hall, 2002, pp. 29-30)

Consideramos que cuando Hall hace referencia a esta doble conciencia, lo hace con la intención de remarcar la manera como los individuos viven cotidianamente las limitaciones de cualquier y toda cultura de proveer por sí misma los significados relevantes para el completo entendimiento de uno mismo, para configurar un sentido de pertenencia unívoco; por lo tanto, está asociada con la doble demanda de igualdad y diferencia. Al mismo tiempo, el llamado por la diferencia parecería apuntar a la dimensión de la agencia, en el sentido de que los individuos intervienen con su intención en la producción de sus identificaciones; sin embargo, qué entiende Hall por 'doble conciencia' es una cuestión que no está clara en su planteamiento del cosmopolitismo vernáculo. ¿Acaso se refiere a la simultaneidad de identificaciones, alineaciones identitarias, suturas, operando simultáneamente? Para nuestros propósitos, revisamos en otras obras de Hall sus reflexiones sobre la constitución de las identidades y cómo estas se construyen en y a través de la 'diferencia'.

\section{Las 'identificaciones' en Hall: las diferencias en las identidades}

En “Introducción: ¿quién necesita 'identidad'"? (1996), Hall retoma e intenta 
responder algunas preguntas formuladas por Avtar Brah (1992), quien cuestiona las nuevas maneras de conceptualizar la identidad, por ejemplo: ¿cómo debe analizarse la subjetividad poscolonial 'racializada' y de género? ¿Cómo se articulan el "orden simbóli-

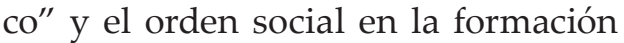
del sujeto? (Hall, 1996, p. 20). Retomando a Foucault, Hall recuerda, por una parte, al sujeto descentrado que se construye discursivamente y que no puede hallarse al margen de las fuerzas regulatorias de la norma; pero aclara que el mismo Foucault demuestra a partir de las 'tecnologías del yo' las prácticas de autoproducción en modos específicos de conducta, o lo que se conoce como performatividad (Hall, 1996, pp. 27-32).

Creo que podemos ver aquí, entonces, que el rigor escrupuloso de su pensamiento empuja a Foucault, a través de una serie de cambios conceptuales en diferentes etapas de su obra, a admitir que, como el descentramiento del sujeto no es su destrucción y el "centramiento" de la práctica discursiva no puede funcionar sin la constitución de sujetos, el trabajo teórico no puede cumplirse plenamente sin complementar la descripción de la regulación discursiva y disciplinaria con una descripción de las prácticas de la autoconstitución subjetiva. (Hall, 1996, p. 32)

Acerca de este mismo punto, Hall encuentra que Judith Butler ha realizado un aporte significativo sobre el entendimiento de las transacciones complejas entre el sujeto, el cuerpo y la identidad, al identificar al sexo como uno de esos principios normativos que Foucault denominó "ideal regulatorio", cuya performatividad (poder que se traduce en prácticas) produce los cuerpos que gobierna. En otras palabras, según Hall, Butler replantea la materialización como un efecto de poder, estableciendo una conexión entre asumir un sexo con la cuestión de la identificación y los medios discursivos por los cuales el imperativo de la normatividad heterosexual legitima ciertas identificaciones sexuadas y rechaza otras (Butler, 1993, en Hall, 1996, p. 34).

Butler argumenta con vigor que todas las identidades actúan por medio de la exclusión, a través de la construcción discursiva de un afuera constitutivo y la producción de sujetos abyectos y marginados, aparentemente al margen del campo de lo simbólico, lo representable -“la producción de un 'afuera', un dominio de efectos inteligibles" (Butler 1993)-, que luego retorna para trastornar y perturbar las exclusiones prematuramente llamadas "identidades". (Hall, 1996, p. 35)

Por esta vía, Butler sostiene que lo que ocurre con la política identitaria feminista, y con todas las otras identidades tratadas políticamente de una manera fundacional, es que en pos de una política representacional, cuya base es la presunta universalidad y unidad, se termina con la producción de sujetos abyectos y marginados por efecto de un 'orden dicotómico compulsivo' (Butler, 1990, en Guerrero, 
1998, p. 114), como puede ser el caso de mujeres 'diferentes' que no caben dentro de la 'normalidad' de las relaciones heterosexuales (Souter, 1995, en Hall, 1996, pp. 35-36, énfasis nuestro).

Un argumento similar presenta Andrés Guerrero para el caso de las identidades racializadas en sociedades poscoloniales como la ecuatoriana. Apoyándose en las ideas de Judith Butler, él sostiene que ha operado una división dual asociada a una matriz binaria de percepción mental propia del sistema colonial de dominación: "La frontera étnica sería una suerte de artilugio simbólico de dominación que, en las relaciones de poder cotidianas, produce y reproduce a la vez al indio y al blanco-mestizo" (Guerrero, 1998, p. 114).

Pero volviendo a Hall y nuestro interés por saber cómo trata la cuestión de la différance y la tensión de la diferencia en la identidad, un dato importante es que desde su posestructuralismo y su propia práctica de deconstrucción, él considera que las nociones que parecen desafiar el binarismo, tales como hibridez, sincretismo, tercer espacio, $\mathrm{u}$ otras, ocultan la producción y reproducción de las estructuras de poder dominantes. Es decir, no hay un 'en medio' que resuelva el problema del poder ni la diferencia. Para este autor no se trata de escapar del binarismo o negarlo, puesto que el binarismo es en realidad una diferencia sobredeterminada, que hace que el poder siempre siga manteniendo los binarios en las realidades históricas:
Esto es posible debido a que el lenguaje y el poder no son lo mismo, sino que el poder interviene en el lenguaje (representación) para asegurar ciertos efectos (...) Esto no hace que la deconstrucción sea inútil (...) solo necesaria pero no suficiente. (Hall en Grossberg, 2006, p. 52)

Por otra parte, en sus reflexiones sobre racismo, partiendo de una rigurosa especificidad histórica, Hall define este fenómeno como una forma o una serie de formas históricamente cambiantes de dividir y distribuir la población, y advierte contra la extrapolación de una estructura esencial racista común para todo caso o incluso suponer que un tipo de racismo se mantiene en el tiempo...

(...) -como una serie de prácticas políticas e ideológicas, de una especie diferenciada- articulada de manera concreta con otras prácticas en una formación social (...) La cuestión no es si los hombres en general establecen diferencias perceptuales entre grupos con diferentes características raciales o étnicas, sino más bien, cuáles son las condiciones específicas que hacen de esta forma de diferenciación algo pertinente socialmente y activo históricamente. (Hall, 1980, en Grossberg, 2006, p. 52)

Por tanto, desde el análisis contextual, Hall apunta a la contingencia de las prácticas discursivas que han tendido a marcar las fronteras de las identidades como algo que también está en constante desplazamiento en términos históricos, pues distingue distintos momentos de identidad étnica o 'tipos de política de la diferencia 
etnitizada' que resultan de arreglos temporales e inestables.

De ahí que la forma de reconciliar la pertenencia y la diferencia, la particularidad y la libertad está para Hall en una especie de interioridad. Este sería el sentido de la identidad que se desprende del cosmopolitismo vernáculo: "El derecho por ejemplo de vivir la propia vida desde el interior -no como se impone o estipula simplemente desde afuera- sino desde adentro, darle una especie de autenticidad desde adentro" (Hall, 2000 en Grossberg, 2006, p. 60). Al mismo tiempo, entendemos que la apelación a la pertenencia como un universal concreto, como condición de posibilidad de la libertad alerta a Hall sobre el retorno del universalismo liberal.

Siguiendo esta argumentación, el cosmopolitismo vernáculo de Hall apunta hacia el ejercicio estratégico de identidades políticas atentas al contexto y la coyuntura como identificaciones o puntos de sutura desde el interior (la conciencia); es decir, unas prácticas comunes, naturalizadas, según las cuales los sujetos son capaces de cuestionar las políticas identitarias dominantes, tanto las que provienen del poder gubernamental como las que pretende imponer la comunidad.

\section{La mirada interseccional sobre la pertenencia y las identidades}

Si bien con Hall podemos entender la noción de identidad como una cuestión histórica, compleja y contradictoria, en proceso continuo, en permanente construcción en y por representaciones (relatos del sí mismo) relacionados con identificaciones en el sentido de suturas o adhesiones temporales, para él no todas las identidades son culturales, no existe una relación garantizada entre las identidades políticas, económicas y culturales. De ahí que, de acuerdo a su planteamiento de política de identidades múltiples, creemos que no se podría pensar en una representación, sutura o identificación, que simultáneamente una varios clivajes sociales distintos, por ejemplo: pertenencia política, género y clase.

En esta parte queremos articular las nociones de pertenencia e identidad con la idea de múltiples escalas y niveles de opresión en interrelación. Consideramos que la relevancia de la 'interseccionalidad' está en que plantea que los diferentes ejes de estratificación se construyen mutuamente los unos a los otros, de manera que las desigualdades están articuladas y conectadas a las diferencias (Roth, 2013; Anthias, 2008). Nira Yuval-Davis, por ejemplo, sostiene que la interseccionalidad es el acercamiento de mayor validez para analizar la estratificación social de manera integral, pues no prioriza una faceta o categoría de diferenciación social, sino que las considera a todas mutuamente constitutivas (Yuval-Davis, 2011, p. 4).

Sobre la pregunta de cómo entender la pertenencia desde la perspectiva interseccional, esta autora plantea que se trata de un proceso siempre di- 
námico - contrario a la noción de fijeza reificada-, pero principalmente considerado como una construcción multinivel, multiescalar y multiterritorial; es decir, en términos analíticos, se puede entender la pertenencia social y política operando en diferentes terrenos o facetas, tales como posiciones o lugares sociales, identificaciones o apegos emocionales a diversas colectividades y agrupaciones, o en relación con los sistemas de valores éticos y políticos (Yuval-Davis, 2011, p. 5).

En este punto, es importante precisar una distinción entre la pertenencia y la identidad. Para Floya Anthias (2008, p. 8), mientras el aspecto clave en la identidad es la articulación entre narrativas individuales y colectivas del sí mismo y del otro, y remite a la estima, la clasificación, mitos de origen y mitos de destino sobre los que operan estrategias de identificación; en la pertenencia es más importante la dimensión experiencial y está relacionada con nociones de exclusión, inclusión, acceso y participación. Entonces, se trata de experiencias formales e informales de pertenencia y la manera como se concibe el apego hacia ciertos espacios sociales construidos e imaginados.

Aunque Stuart Hall no se posiciona desde la interseccionalidad, su noción de identidad implica una manera similar de entender la pertenencia:

La identificación se construye sobre la base del reconocimiento de algún origen común o unas características compartidas con otra persona o grupo o con un ideal, y con el vallado natural de la solidaridad y la lealtad establecidas sobre este fundamento. (Hall, 1996, p. 15)

Entonces, si bien identidad y pertenencia se construyen en términos relacionales e intersubjetivos, Anthias remarca que la diferencia entre estas dos nociones está en el énfasis sobre la vivencia del lazo; por un lado, la identidad involucra narrativas individuales y colectivas, es decir, representaciones de nosotros mismos (y de los otros); por otro lado, la pertenencia consiste en las experiencias de ser parte de una comunidad y las maneras en las que los lazos sociales se manifiestan en prácticas, experiencias y emociones de inclusión.

Pero más que eso, sobre la cuestión de la pertenencia desde la mirada de la interseccionalidad, la autora sostiene que, por ejemplo, los lazos étnicos no podrían ser considerados por separado como proporcionando 'pertenencia' sin tomar en cuenta que estos mismos lazos están intersectados por diferentes tipos de relaciones sociales como los de clase, generacionales, de género; por lo tanto, pertenecer es ser aceptado en una comunidad y estos lugares comunes están ellos mismos atravesados por diversas posiciones, divisiones sociales y clivajes (Anthias, 2008, p. 8).

Hasta aquí, consideramos que la perspectiva de la interseccionalidad apunta a entender los sentidos múltiples de la pertenencia y la configuración de las identidades desancladas de estructuras fijas, pero -más que eso- constituidas en torno a la con- 
frontación con diversas divisiones sociales y estructuras de desigualdad operando simultáneamente.

Ante la pregunta de cómo estas estructuras de desigualdad son tratadas en articulación con la pertenencia y las identidades, Anthias responde que hay distintos énfasis en el uso de la interseccionalidad. Su posición, junto con la de Yuval-Davis, es que las divisiones sociales se refieren a ontologías sociales en torno a diferentes procesos materiales, todos articulados a la sociabilidad y a la organización social de la sexualidad, la producción y los lazos colectivos, dimensiones que pueden estar en todas las sociedades (Anthias, 2008, p. 13). Sin embargo, la autora advierte:

Un peligro con la noción de intersecciones tiene que ver con pensar a las personas como perteneciendo a grupos fijos y permanentes (étnicos, de género o clase, por ejemplo), los cuales luego caen en un determinismo de estilo pluralista. Esto socava el propósito de enfocarse en los procesos sociales, las prácticas y los resultados, la manera cómo impactan en las categorías, las estructuras sociales e individuales. (Anthias, 2008, p. 14, énfasis original)

Si tomamos en cuenta que los grupos son importantes en dos sentidos: en términos de los atributos de ser parte y lo que estos atributos implican, y en cuanto a las formas de encuentro y participación social, entonces el núcleo de la interseccionalidad es relevante para la teorización de las identidades. Los clivajes etnicidad/nación, sexo/género, clase, nacionalidad u otros involucran distintas y variables formas de articulación social e identificaciones que se mueven de una manera altamente matizada y contextual (Anthias, 2008, p. 14).

Consiguientemente, a pesar de la dificultad que implica pensar en clave interseccional porque esta perspectiva rechaza la idea de posiciones políticas fijas asociadas a una forma singular de identidad y -en cambio- reconoce una multiplicidad de subcategorías y formas entrecruzadas, consideramos que puede constituirse en un andamiaje más integral, que finalmente permita la articulación entre las diferencias, las desigualdades sociales y las identidades múltiples (Anthias, 2008, p. 14). A continuación revisamos el planteamiento de Anthias sobre una herramienta heurística que puede contribuir a este propósito.

\section{Posicionamiento translocacional}

Con la idea del posicionamiento translocacional, Anthias quiere evocar la imagen de los interminables movimientos de las personas tanto en términos físicos como entre dimensiones sociales y de sentido. Partiendo de la premisa de que nuestras localizaciones son múltiples y tocan una diversidad de terrenos, como aquellos de género, clase, etnicidad y nación, sistemas políticos y de valoración, ella sostiene que es fundamental considerar los vínculos entre las relaciones sociales y particularmente aquellas que pro- 
ducen estructuras de diferenciación e identificación y estructuras de exclusión e inclusión, porque en términos de la jerarquía social una persona puede estar localizada en una posición diferente, dependiendo de la relevancia de una determinada categoría, ya sea en términos contextuales, temporales o de sentido, y en relación a diferentes prácticas regulatorias del Estado, así como en términos del entendimiento de la misma persona acerca de su posición social (Anthias, 2008).

Esta noción, de acuerdo con Anthias, ayuda a pensar la vida como transcurriendo en el cruce de múltiples $y$, también, fragmentados espacios sociales que se interrelacionan.

Pueden ser pensados como espacios sociales definidos por fronteras por un lado, y por jerarquías, por el otro. La cuestión con las fronteras y jerarquías está en el centro del concepto. Se aleja particularmente de la idea de atravesar grupos que caracteriza mucho de la discusión de la interseccionalidad. (Anthias, 2008, p. 15)

Por su parte, la idea de posicionamiento alude a la intersección entre 'estructura', en términos de un conjunto de efectos y efectividades sociales que señalan una posición social, y 'agencia', entendida como un conjunto de prácticas, acciones y sentidos, en cuanto procesos por los que alguien se posiciona socialmente. Mientras que la noción de localización remarca la importancia del contexto, la naturaleza situada de las demandas y atribuciones, la varia- bilidad de los procesos (Anthias, 2008, p. 15, énfasis nuestro).

El término translocacional se refiere a la naturaleza compleja del posicionamiento de aquellos quienes se ubican en el encuentro de una gama de locaciones y dislocaciones en relación con género, etnicidad, pertenencia nacional, clase y racialización. El posicionamiento toma lugar en el contexto de prácticas vividas en que la identificación es practicada/performada, así como las condiciones intersubjetivas, organizacionales y de representación que las hacen posibles. (Anthias, 2002, en Anthias, 2008, pp. 15-16)

Anthias considera que este lente analítico puede ayudar en el abordaje de identidades múltiples e identidades intersectadas bajo la consideración de tres elementos. Primero, tanto 'diferencia' como 'identidad' se conciben como conjuntos de procesos materiales y culturales, no como atributos o características que los individuos poseen; de acuerdo con esta autora, las personas producen su identidad en la interacción con regímenes normativos que despliegan narrativas y prácticas hegemónicas, pero también como recursos para la acción social. Segundo, este abordaje se aleja de la idea de examinar transversalmente grupos sociales o categorías y privilegia un análisis de procesos sociales más amplios en el contexto espacial y temporal a partir de la interpelación a diferentes discursos, prácticas y estructuras en un nivel macro. Tercero, esto puede visibilizar cómo las divisiones sociales se articu- 
lan para producir una ampliación de prácticas de subordinación, o conducir hacia procesos altamente contradictorios en la interacción de diferentes discursos, prácticas y regímenes regulatorios, con implicaciones en la producción de formas de posicionamiento e identidad (Anthias, 2008, p. 16).

\section{Reflexiones finales}

$\mathrm{Si}$, como hemos revisado a través de los postulados, para Stuart Hall las identidades son -por un lado- puntos de encuentro y de sutura entre discursos y prácticas que interpelan al yo, y -por otro lado- hacen referencia a la autenticidad (cierre) producida desde la interioridad; es decir, si son alineaciones entre dos ámbitos de creación de sentido: un afuera constitutivo y una interioridad de producción del sujeto (Butler, 1993, en Hall, 1996, p. 36), nos preguntamos si es posible pensar -dentro el esquema de Hall- en la actualización (sutura) de múltiples identificaciones simultáneamente interpeladas por discursos que aluden a distintos ejes de diferenciación social. ¿Es posible pensar más allá de las identidades múltiples, en múltiples identidades operando simultáneamente sin efectos devastadores de contradicción interna; es decir, sin que implique la invalidación de unas respecto de otras? ¿Logra resolver esta cuestión la noción de uso estratégico de las identidades?

En un primer acercamiento a estas preguntas, consideramos que la alusión de Hall a una doble conciencia en cuanto a la pertenencia política en contextos transnacionales apunta a este uso estratégico de identidades y lealtades políticas en tensión. Pero de acuerdo a su comprensión de la identidad en términos generales, más allá de la pertenencia a diversas comunidades, consideramos que este autor no ha logrado resolver la cuestión de la identidad en articulación con los diferentes ejes de estratificación: género, clase, nacionalidad, étnica y otras.

Frente a estos temas, la propuesta de posicionamiento translocacional de Floya Anthias plantea una manera diferente de analizar las identidades que pueden permitir estas articulaciones. Partiendo de la perspectiva interseccional, que considera que los ejes de estratificación están interconectadamente constituidos los unos a los otros, Anthias sostiene que las identidades se constituyen no en relación a grupos fijos, como pueden ser las representaciones de clase, etnia, nación, etc., sino a espacios sociales (construidos o imaginarios) en torno a narrativas hegemónicas, interactuando con sentidos y prácticas como recursos de acción social.

De ahí que nos parece significativo el aporte de pensar las identidades no como puntos de sutura o esfuerzos de alineación, sino como posicionamientos espacio-temporales, sociales y de sentido, locaciones que no son fijas sino que son relacionales en el contexto y el tiempo; por lo tanto involucran cambios y contradicciones. 
Consideramos que esta es una manera vernácula de articular las identidades en la práctica y la experiencia, pues entiende que si bien las identidades se actualizan de manera estratégica, también permite representar las múltiples identificaciones operando simultáneamente, sin que la visibilidad de alguna de ellas (identidad de clase, por ejemplo) suponga la desactivación o anulación de las demás (identidades de género, étnica, nacional, etc.), sino solo una predominancia en términos contextuales y coyunturales.

\section{Referencias}

Anthias, F. (2008). Thinking through the lens of translocational positionality: an intersectionality frame for understanding identity and belonging. En Translocations: Migration and Social Change. An Inter-Disciplinary Open Access E-Journal, 4, 5-20.

Brah, A. 1992. Diference, diversity and differentiation. En J. Ronald y A. Rattansi (Eds.), Race, culture and difference, pp. 126-145. Londres: Sage.

Grossberg, L. (Diciembre de 2006). Stuart Hall sobre raza y racismo: estudios culturales y la práctica del contextualismo. Tabula Rasa 5, 45-65. Bogotá: Universidad Colegio Mayor de Cundinamarca.
Guerrero, A. (diciembre de 1997). Ciudadanía, frontera étnica y compulsión binaria. Íconos, 4, 112-122. Quito: Flacso.

Hall, S. (1996). Introducción: ¿quién necesita "identidad"? En S. Hall y P. Du Gay (Coords.), Cuestiones de identidad cultural, pp. 13-39. Madrid: Amorrotu Editores.

Hall, S. (2002). Political belonging in a world of multiple identities. En S. Vertovec y R. Cohen (Coords.), Conceiving cosmopolitanism. Theory, context and practice, pp. 25-32. Londres, Nueva York: Oxford University Press.

Roth, J. (2013). Entangled inequalities as intersectionalities: towards an epistemic sensibilization. desiguALdades.net Working Paper Series, 43. Berlin: desiguALdades.net Research Network on Interdependent Inequalities in Latin America. Recuperado de http://www.desigualdades.net/ Resources/Working_Paper/43_WP_ Roth_Online.pdf

Yuval-Davis, N. (2011). Power, intersectionality and the politics of belonging. Feminist Research Center in Aalborg. FREIA Working Paper Series, 75. Aalborg: Aalborg Universitet, Institut for Kultur og Globale Studier. 